\title{
The biogenesis of platelets from megakaryocyte proplatelets
}

\author{
Sunita R. Patel, John H. Hartwig, and Joseph E. Italiano Jr.
}

Hematology Division, Department of Medicine, Brigham and Women's Hospital, Boston, Massachusetts, USA.

\begin{abstract}
Platelets are formed and released into the bloodstream by precursor cells called megakaryocytes that reside within the bone marrow. The production of platelets by megakaryocytes requires an intricate series of remodeling events that result in the release of thousands of platelets from a single megakaryocyte. Abnormalities in this process can result in clinically significant disorders. Thrombocytopenia (platelet counts less than $150,000 / \mu 1$ ) can lead to inadequate clot formation and increased risk of bleeding, while thrombocythemia (platelet counts greater than $600,000 / \mu 1)$ can heighten the risk for thrombotic events, including stroke, peripheral ischemia, and myocardial infarction. This Review will describe the process of platelet assembly in detail and discuss several disorders that affect platelet production.
\end{abstract}

\section{Platelet formation}

Megakaryocyte development. Megakaryocytes are rare myeloid cells (constituting less than $1 \%$ of these cells) that reside primarily in the bone marrow (1) but are also found in the lung and peripheral blood. In early development, before the marrow cavities have enlarged sufficiently to support blood cell development, megakaryopoiesis occurs within the fetal liver and yolk sac. Megakaryocytes arise from pluripotent HSCs that develop into 2 types of precursors, burst-forming cells and colony-forming cells, both of which express the CD34 antigen (2). Development of both cell types continues along an increasingly restricted lineage culminating in the formation of megakaryocyte precursors that develop into megakaryocytes (1). Thrombopoietin (TPO), the primary regulator of thrombopoiesis, is currently the only known cytokine required for megakaryocytes to maintain a constant platelet mass (3). TPO is thought to act in conjunction with other factors, including IL-3, IL-6, and IL-11, although these cytokines are not essential for megakaryocyte maturation (4).

Megakaryocytes tailor their cytoplasm and membrane systems for platelet biogenesis. Before a megakaryocyte has the capacity to release platelets, it enlarges considerably to an approximate diameter of $100 \mu \mathrm{m}$ and fills with high concentrations of ribosomes that facilitate the production of platelet-specific proteins (5). Cellular enlargement is mediated by multiple rounds of endomitosis, a process that amplifies the DNA by as much as 64-fold (6-9). $\mathrm{TPO}$, which binds to the c-Mpl receptor, promotes megakaryocyte endomitosis. During endomitosis, chromosomes replicate and the nuclear envelope breaks down. Although interconnected mitotic spindles assemble, the normal mitotic cycle is arrested during anaphase B. The spindles fail to separate, and both telophase and cytokinesis are bypassed. Nuclear envelope reformation $(10,11)$ results in a polyploid, multilobed nucleus with DNA contents ranging from $4 \mathrm{~N}$ up to $128 \mathrm{~N}$ within each megakaryocyte (12).

In addition to expansion of DNA, megakaryocytes experience significant maturation as internal membrane systems, granules, and

Nonstandard abbreviations used: DMS, demarcation membrane system; GP, glycoprotein; PKC $\alpha$, protein kinase C $\alpha$; TPO, thrombopoietin.

Conflict of interest: The authors have declared that no conflict of interest exists.

Citation for this article: J. Clin. Invest. 115:3348-3354 (2005).

doi:10.1172/JCI26891. organelles are assembled in bulk during their development. In particular, there is the formation of an expansive and interconnected membranous network of cisternae and tubules, called the demarcation membrane system (DMS), which was originally thought to divide the megakaryocyte cytoplasm into small fields where individual platelets would assemble and subsequently release (13). DMS membranes have continuity with the plasma membrane $(14,15)$ and are now thought to function primarily as a membrane reservoir for the formation of proplatelets, the precursors of platelets. A dense tubular network (16) and the open canalicular system, a channeled system for granule release, are also formed before the assembly of proplatelets begins. Specific proteins associated with platelets, such as vWF and fibrinogen receptors, are synthesized and sent to the megakaryocyte surface, while others are packaged into secretory granules with such factors as vWF, which is loaded into $\alpha$-granules (17). Still other proteins, such as fibrinogen, are collected from plasma through endocytosis and/or pinocytosis by megakaryocytes and are selectively placed in platelet-specific granules $(17,18)$. Also assembled during megakaryocyte maturation are mitochondria and dense granules, which, like $\alpha$-granules, derive from Golgi complexes. Thus, as terminally differentiated megakaryocytes complete maturation, they are fully equipped with the elements and machinery required for the major task of platelet biogenesis.

The flow model of platelet formation. Despite the identification of platelets over 120 years ago, there is still little consensus on many of the mechanisms involved in platelet biogenesis. However, recent evidence supports a modified flow model of platelet assembly. In this model, platelets are assembled along essential intermediate pseudopodial extensions, called proplatelets, generated by the outflow and evagination of the extensive internal membrane system of the mature megakaryocyte (19). In 1906, Wright introduced the initial concept that platelets arise from megakaryocyte extensions when he described the detachment of platelets from megakaryocyte pseudopods (20). Almost a century later, studies on megakaryocytes producing platelets in vitro have revealed the details of platelet assembly and have led us back to the classical proplatelet theory of platelet release in which platelets fragment from the ends of megakaryocyte extensions (21-23). The discovery and cloning of TPO in 1994 and its receptor, $\mathrm{c}-\mathrm{Mpl}$, have allowed major advances in the study of thrombopoiesis (24). TPO has facilitated the development of in vitro megakaryocyte culture systems through which the pro- 


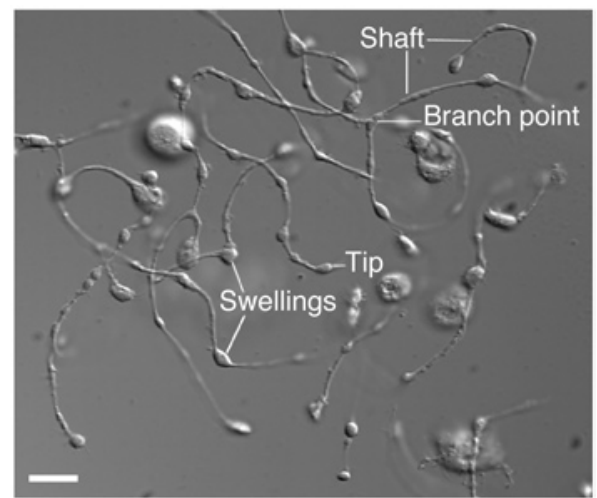

\section{Figure 1}

Anatomy of a proplatelet. Differential interference contrast image of proplatelets on a mouse megakaryocyte in vitro. Some of the hallmark features of proplatelets, including the tip, swellings, shafts, and a branch point, are indicated. Scale bar, $5 \mu \mathrm{m}$.

cess of platelet formation can be directly visualized and analyzed (25-29). These systems have successfully reconstituted the transition of terminally differentiated megakaryocytes into fully functional platelets. Megakaryocytes cultured in the presence of TPO extend numerous proplatelets, consistent with the flow model. The proplatelets generated in the in vitro systems are structurally similar to those seen in vivo extending into bone marrow sinusoids and within the bloodstream (30-32). Significantly, platelets released from megakaryocytes in vitro are structurally and functionally similar to those found in vivo $(28,29)$. They are discs, $2-3 \mu \mathrm{m}$ in diameter, that contain a marginal microtubule band, and change shape in response to platelet agonists, including thrombin.

Transcriptional control of platelet formation. A complete understanding of platelet formation will rely heavily on the identification of cellular controls active at each step of the elaborate process described above. To date, only a few transcription and signaling factors have been implicated in platelet generation, in part because of the rarity of megakaryocytes in bone marrow.

GATA- 1 and FOG (friend of GATA) are 2 transcription factors with major roles in thrombopoiesis. GATA-1 acts early in megakaryocyte development, where it is involved in lineage commitment of megakaryocytes, as well as erythrocytes, from their respective progenitor cells (33). GATA-1 also functions later in megakaryocyte development, controlling proliferation. Mutant mice that fail to accumulate GATA-1 within their megakaryocytes exhibit thrombocytopenia and possess an increased number of immature megakaryocytes within their bone marrow. These megakaryocytes exhibit small size, underdeveloped DMSs, reduced platelet-specific granule content, decreased polyploidization, and an excess of rough endoplasmic reticulum (34). In humans, missense mutations in GATA-1 that disrupt its interaction with FOG-1 lead to thrombocytopenia and abnormal bone marrow megakaryocytes. A truncated version of GATA-1, expressed in transient myeloproliferative disorder and acute megakaryoblastic leukemia, is able to interact with FOG-1 but lacks an N-terminal activation domain, resulting in decreased transcriptional activation (35). Thus GATA-1 and FOG-1 appear to play critical roles in megakaryocyte maturation.

The transcription factor NF-E2 has been identified as a major regulator of platelet biosynthesis. NF-E2 is a heterodimer of p45 and $\mathrm{p} 18$ subunits that assumes the basic leucine zipper motif (36). NF-E2 null mice experience lethal thrombocytopenia and die from hemorrhage since they lack circulating platelets (37). Megakaryocytes from NF-E2-null mice fail to undergo proplatelet formation, although megakaryocyte maturation appears intact (38). Interestingly, megakaryocytes from NF-E2 knockout mice lack $\beta_{1}$-tubulin, the major $\beta$-tubulin isoform expressed in megakaryocytes and platelets (39). NF-E2 has also been shown to interact with the promoter for Rab27b, a small GTPase identified in platelets. Rab27b expression is high in terminally differentiated megakaryocytes, and its inhibition in megakaryocytes results in attenuated proplatelet production, which suggests a role for Rab27b in proplatelet formation (40). NF-E2 may also affect thromboxane synthase (41) and caspase-12 (42), both of which are reduced in NF-E2-null megakaryocytes.

Overview of proplatelet formation. The assembly of platelets from megakaryocytes involves an elaborate dance that converts the cytoplasm into 100 - to $500-\mu \mathrm{m}$-long branched proplatelets on which the individual platelets develop. The proplatelet and platelet formation process generally commences from a single site on the megakaryocyte where 1 or more broad pseudopodia form. Over a period of 4-10 hours, the pseudopodial processes continue to elongate and become tapered into proplatelets with an average diameter of 2-4 $\mu \mathrm{m}$. Proplatelets are randomly decorated with multiple bulges or swellings, each similar in size to a platelet, which gives them the appearance of beads connected by thin cytoplasmic strings (Figure 1). The generation of additional proplatelets continues at or near the original site of proplatelet formation and spreads in a wavelike fashion throughout the remainder of the cell until the megakaryocyte cytoplasm is entirely transformed into an extensive and complex network of interconnected proplatelets $(27,36)$. The multilobed nucleus of the megakaryocyte cell body is compressed into a central mass with little cytoplasm and is eventually extruded and degraded. Platelet-sized swellings also develop at the proplatelet ends and are the primary sites of platelet assembly and release, as opposed to the swellings along the length of the proplatelet shaft (Figure 1). The precise events involved in platelet release from proplatelet ends have not been identified.

Microtubule organization in proplatelets. Microtubules, hollow polymers assembled from $\alpha \beta$-tubulin dimers, are the major structural component of the engine that drives the elongation of proplatelets. It is well known that microtubule bundles assembled by the megakaryocytes fill proplatelet processes $(27,43,44)$, as shown in Figure 2. Likewise, when megakaryocytes are retrovirally directed to express GFP-tagged $\beta_{1}$-tubulin, fluorescent microtubules densely fill their proplatelets (45). The microtubules arrays are essential for proplatelet formation (26). Proplatelets fail to form in megakaryocytes treated with agents that inhibit microtubule assembly (1-10 $\mu \mathrm{m}$ nocodazole) $(26,27,46)$. Transgenic mice lacking $\beta_{1}$-tubulin, the most abundant platelet $\beta$-tubulin isoform, assemble microtubules poorly, develop thrombocytopenia (15-30\% reduction in platelet count), and have spherocytic circulating platelets, a consequence of defective marginal band formation (44). The marginal bands of $\beta_{1}$-tubulin-null platelets are composed of a reduced microtubule mass and have only $2-3$ coilings instead of the normal 8-12. The importance of $\beta_{1}$-tubulin in platelet-shape maintenance is supported by a recent study that identified the first $\beta_{1}$-tubulin variation in humans, where a double-nucleotide mutation results in the substitution of a highly conserved glutamine with a proline (Q43P) (47). In heterozygous individuals carrying the Q43P mutation, 

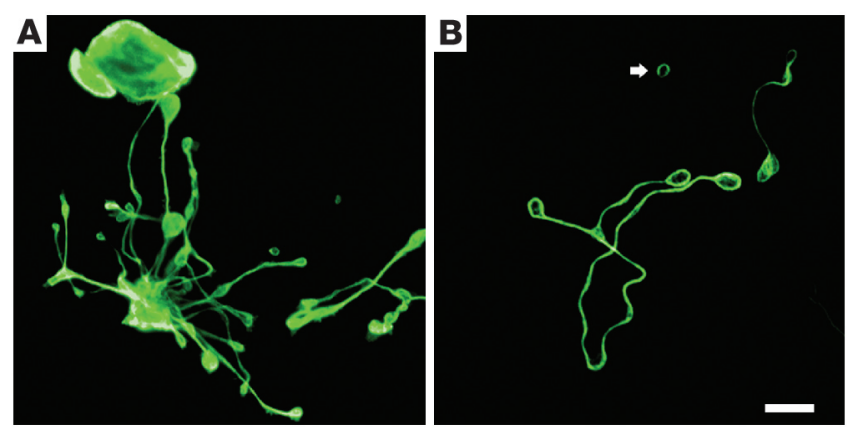

Figure 2

Localization of microtubules within proplatelets. (A) Immunofluorescence studies on murine megakaryocytes grown in culture and labeled with $\beta_{1}$-tubulin antibodies indicate that microtubules line the entire length of proplatelets, including shafts and the tip. (B) Immunofluorescence studies further show that microtubule coils similar to those seen in mature platelets occur in both proplatelets and released platelet-sized particles (arrow). Scale bar, $5 \mu \mathrm{m}$.

$\beta_{1}$-tubulin expression was reduced in platelets, which were enlarged and spherocytic because of defects in the microtubule marginal band. Additionally, the Q43P mutation is thought to occur quite frequently in the normal population $(-10 \%)$ and impart a protective effect against cardiovascular disease in men (47).

Electron microscopy studies of megakaryocytes undergoing proplatelet formation have provided insights into how microtubule reorganization powers proplatelet growth (27). Just before proplatelet formation, microtubules consolidate in a mass just beneath the cortical plasma membrane. These microtubules align into bundles and fill the cortex of the first blunt process extended by megakaryocytes, signaling the beginning of proplatelet development. The microtubules merge into thick linear bundles that fill the proplatelet shafts when the proplatelets lengthen and taper. At the free proplatelet end, the microtubule bundles form loops, which reenter the proplatelet shaft. This process gives rise to the bulbous tips of the proplatelets, each measuring 3-5 $\mu \mathrm{m}$ in diameter. These studies also were the first to recognize that the plateletsized swellings that occur along proplatelet shafts are not nascent platelets but are instead points where the microtubule bundles of the shaft diverge for a short distance and then reconvene to locally thicken the proplatelet shaft. Coiling of microtubules, the signature of circulating platelets, occurs only at the proplatelet ends and not within the platelet-sized swelling positioned along the proplatelet shaft. Therefore, the primary site of platelet assembly is at the end of each proplatelet.

Proplatelet elongation. Proplatelets grow from the megakaryocyte cell body at an average rate of $0.85 \mu \mathrm{m} / \mathrm{min}$, in good agreement with the 4-10 hours required to convert the entire megakaryocyte cytoplasm into proplatelets with average lengths of $250-500 \mu \mathrm{m}$ (48). Microtubule assembly dynamics within megakaryocytes and proplatelet formation are complex, and their exact relationship to growth, other than supplying microtubule mass, is unclear. In recent studies, EB3, a protein that binds the plus end of microtubules, fused to GFP was expressed in murine megakaryocytes and used as a marker of microtubule plus-end dynamics. Immature megakaryocytes without proplatelets employ a centrosomalcoupled microtubule nucleation/assembly reaction, which appears as a prominent starburst pattern when visualized with EB3-GFP.
Microtubules assemble only from the centrosomes and grow outward to the cell cortex, where they turn and run in parallel with the cell edges (49). However, just before proplatelet production, centrosomal assembly ceases and microtubules begin to collect in the cell cortex. Once proplatelet extension begins, microtubule nucleation and growth occur continuously throughout the entire proplatelet, including the shaft, swellings, and tip. The EB3-GFP studies also revealed that microtubules polymerize in both directions in proplatelets, e.g., toward both the tips and the cell body (49). This demonstrates that the microtubules composing the bundles have a mixed polarity. The rates of microtubule polymerization are approximately 10 -fold faster than the proplatelet growth rate.

Although microtubules are continuously polymerizing in proplatelets, polymerization per se does not provide the forces for elongation. Proplatelets continue to elongate at normal rates even when microtubule polymerization is inhibited by drugs that block net microtubule assembly, which suggests another mechanism for proplatelet elongation (49). Consistent with this idea, proplatelets possess an inherent microtubule sliding mechanism, similar to the extension of a fire engine ladder. Dynein, a minus-end microtubule molecular motor protein, localizes along the microtubules of the proplatelet and appears to directly contribute to microtubule sliding, since inhibition of dynein, through disassembly of the dynactin complex, prevents proplatelet formation (49). Microtubule sliding can also be reactivated in detergent-permeabilized proplatelets. ATP, known to support the enzymatic activity of microtubule-based molecular motors, activates proplatelet elongation in permeabilized proplatelets (48) that contain both dynein and its regulatory complex, dynactin. Thus, dynein-facilitated microtubule sliding appears to be the key event in driving proplatelet elongation.

Platelet amplification. Each megakaryocyte has been estimated to generate and release thousands of platelets (50-52). If platelet formation is restricted to a relatively limited number of proplatelet ends, platelets would have to form and release on a minute time scale. (The average megakaryocyte has approximately 5-10 original proplatelets. If 1,000 platelets are constructed, then each end would have to produce 100-200 platelets over a 4-hour time course, equivalent to 25-50 platelets per hour.) Analysis of time-lapsed video microscopy of proplatelet development from megakaryocytes grown in vitro, however, has revealed that ends are amplified in an elaborate process that repeatedly bends and bifurcates the proplatelet shaft to form new ends (27). End amplification initiates when a proplatelet shaft is bent into a sharp kink, which then folds back on itself, forming a loop in the microtubule bundle. The new loop eventually elongates, forming a new proplatelet shaft branching from the side of the original proplatelet. Loops lead the proplatelet tip and define the site where nascent platelets will assemble and where platelet-specific contents are trafficked (Figure 3). In marked contrast to the microtubule-based motor that elongates proplatelets, actin-based force is used to bend the proplatelet in end amplification. Megakaryocytes treated with one of the actin toxins cytochalasin and latrunculin can extend long proplatelets but fail to branch and are decorated with few swellings along their length (27). Despite extensive characterization of actin filament dynamics during platelet activation, how actin participates in this reaction and the cytoplasmic signals that regulate bending have yet to be determined. Immunofluorescence and electron microscopy of megakaryocytes undergoing proplatelet formation indicate that actin filaments are distributed throughout the proplatelet and are particularly abundant within swellings and at proplatelet branch 


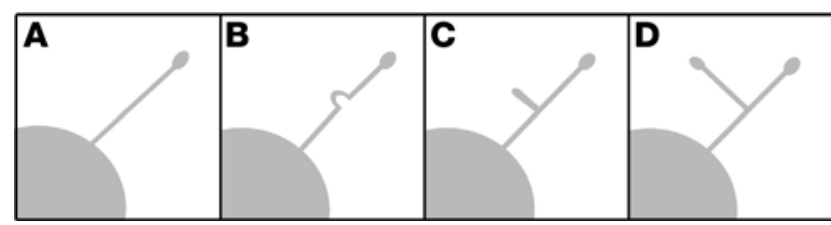

Figure 3

Proplatelet amplification. Megakaryocytes increase their proplatelet number through formation of branched extensions off of existing proplatelets. Initially, the shaft of the parent proplatelet $(\mathbf{A})$ is sharply bent (B). This bend then folds back on itself to form a loop (C). The loop elongates to form a new proplatelet with a novel tip (D).

points $(27,53)$. Studies also indicate that protein kinase $\mathrm{C} \alpha(\mathrm{PKC} \alpha)$ associates with aggregated actin filaments in megakaryocytes undergoing proplatelet formation, and inhibition of $\mathrm{PKC} \alpha$ or integrin signaling pathways prevents actin filament aggregation and proplatelet formation in megakaryocytes (53). However, the role of actin filament dynamics in platelet biogenesis remains unclear.

Organelle transport in proplatelets. Nascent platelets forming at the proplatelet tips must be loaded with their contents of organelles and platelet-specific granules. This process occurs along the shafts of proplatelets, as organelles and granules travel in a discontinuous fashion from cell body to proplatelet. Despite bidirectional movement along shafts, the particles are eventually captured at the proplatelet tip (54). Immunofluorescence and electron microscopy studies indicate that organelles are in direct contact with microtubules (48), and their movement appears to be independent of actin. Of the 2 major microtubule motors, kinesin and dynein, only the plus end-directed kinesin is situated in a pattern similar to that of organelles and granules and is likely responsible for transporting these elements along microtubules (54). It appears that a 2 -fold mechanism of organelle and granule movement occurs in platelet assembly. First, organelles and granules travel along microtubules, and second, the microtubules themselves can slide bidirectionally in relation to other motile filaments to indirectly move organelles along proplatelets in a "piggyback" fashion.

Proplatelet release. In vivo, proplatelets extend into bone marrow vascular sinusoids, where they may be released and enter the bloodstream. The actual events surrounding platelet release in vivo have not been identified because of the rarity of megakaryocytes within the bone marrow. The events leading up to platelet release within cultured murine megakaryocytes have been documented. After complete conversion of the megakaryocyte cytoplasm into a network of proplatelets, a retraction event occurs, which releases individual proplatelets from the proplatelet mass (27). Proplatelets are released as chains of platelet-sized particles, with the most commonly released structure resembling a barbell, a narrow shaft connecting 2 teardrop-shaped tips. Electron microscopic analysis of these barbell structures illustrates linear microtubule bundles within the shafts and a coil of microtubules, reminiscent of the mature platelet marginal band, within the ends. Individual platelet-sized particles are also released from proplatelet-producing megakaryocytes or released proplatelets. Although the actual release event has yet to be captured, the platelet-sized particle is thought be liberated as the proplatelet shaft increasingly narrows. See Figure 4 for an overview of platelet formation.

Apoptosis in platelet biogenesis. The process of platelet assembly in megakaryocytes exhibits some characteristics associated with apoptosis, including cytoskeletal reorganization, membrane con- densation, and ruffling. These similarities have led to further investigations aimed at determining whether apoptosis is a major force driving proplatelet formation and platelet release. Apoptosis, programmed cell death, is responsible for destruction of the nucleus in senescent megakaryocytes (55). However, it is thought that a specialized apoptotic process may lead to platelet assembly and release. Apoptosis has been described in megakaryocytes (56) and found to be more prominent in mature megakaryocytes as opposed to immature cells $(57,58)$. A number of apoptotic factors, both proapoptotic and antiapoptotic, have been identified in megakaryocytes (reviewed in ref. 59). Apoptosis-inhibitory proteins such as $\mathrm{Bcl}-2$ and $\mathrm{Bcl}-\mathrm{x}_{\mathrm{L}}$ are expressed in early megakaryocytes. When overexpressed in megakaryocytes, both factors inhibit proplatelet formation $(60,61)$. Bcl-2 is absent in mature blood platelets, and $\mathrm{Bcl}-\mathrm{x}_{\mathrm{L}}$ is absent from senescent megakaryocytes (62), consistent with a role for apoptosis in mature megakaryocytes. Proapoptotic factors, including caspases and NO, are also expressed in megakaryocytes. Evidence indicating a role for caspases in platelet assembly is strong. Caspase activation has been established as a requirement for proplatelet formation. Caspase- 3 and caspase- 9 are active in mature megakaryocytes, and inhibition of these caspases blocks proplatelet formation (60). NO has been implicated in the release of platelet-sized particles from the megakaryocytic cell line Meg-01 and may work in conjunction with TPO to augment platelet release $(63,64)$. Other proapoptotic factors expressed in megakaryocytes and thought to be involved in platelet production include TGF- $\beta_{1}$ and SMAD proteins (65). Of interest is the distinct accumulation of apoptotic factors in mature megakaryocytes and mature platelets (66). For instance, caspase- 3 and caspase- 9 are active in terminally differentiated megakaryocytes. However, only caspase- 3 is abundant in platelets (67), while caspase- 9 is absent (66). Similarly, caspase-12, found in megakaryocytes, is absent in platelets (42). These data support differential mechanisms for programmed death in platelets and megakaryocytes and suggest the selective delivery and restriction of apoptotic factors to nascent platelets during proplatelet-based platelet assembly.

\section{Disorders of platelet production}

A diversity of factors can contribute to anomalous platelet counts; one of these is inappropriate platelet production. Disorders of inappropriate platelet production are grouped into 2 major categories, inherited and noninherited disorders. Inherited platelet disorders occur in individuals harboring genetic mutations within genes that are active during the process of platelet biogenesis. Identification of the precise genetic lesions that give rise to such disorders gives further insight into the mechanisms of platelet formation. These rare disorders, including the 3 major disorders described below, can result in severe thrombocytopenia and increased bleeding times.

Bernard-Soulier syndrome. Bernard-Soulier syndrome is an autosomal dominant disorder characterized by macrothrombocytopenia (thrombocytopenia with increased platelet volume), increased bleeding time, and impaired platelet agglutination (68). The underlying cause is absent or deficient expression of the glycoprotein (GP) Ib/IX/V complex, which forms the vWF receptor on the platelet surface (69). Binding of vWF to the GPIb/IX/V complex is an essential step in hemostasis. A host of mutations within the genes that encode GPIb $\alpha$, GPIb $\beta$, or GPIX have been identified and linked to Bernard-Soulier syndrome. How these mutations translate into macrothrombocytopenia, however, is still unknown. 
A

B

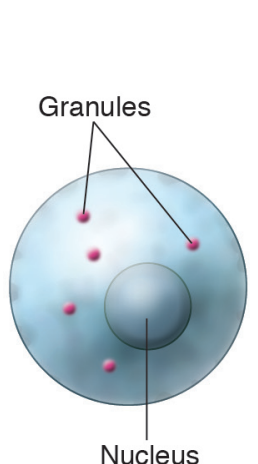

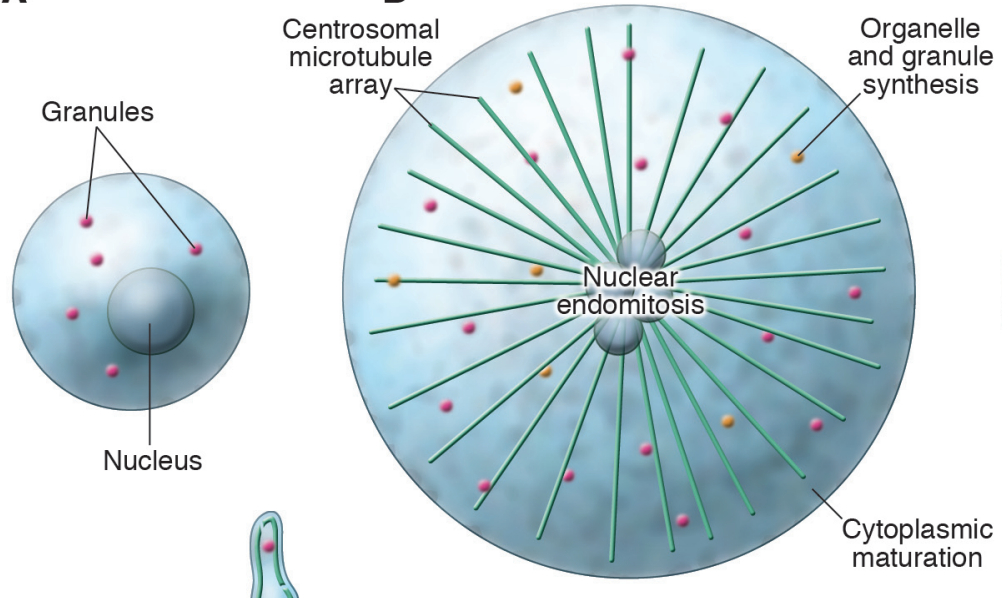

C

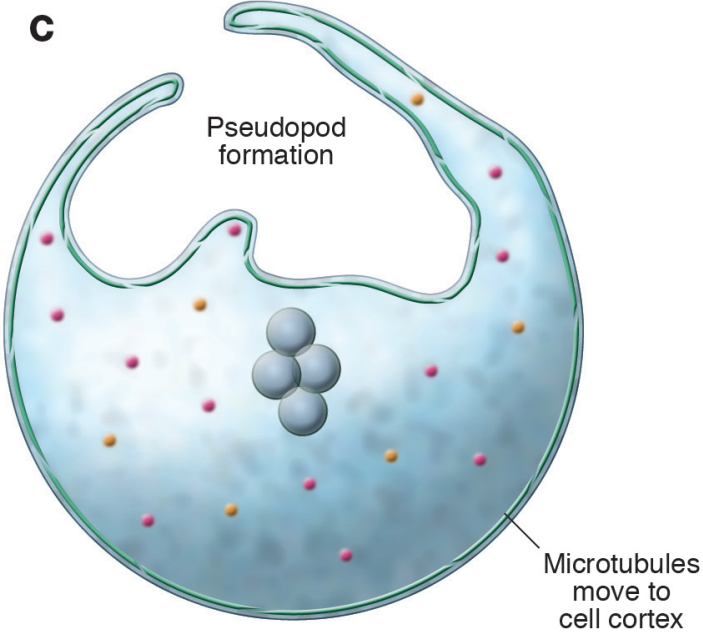
cell cortex

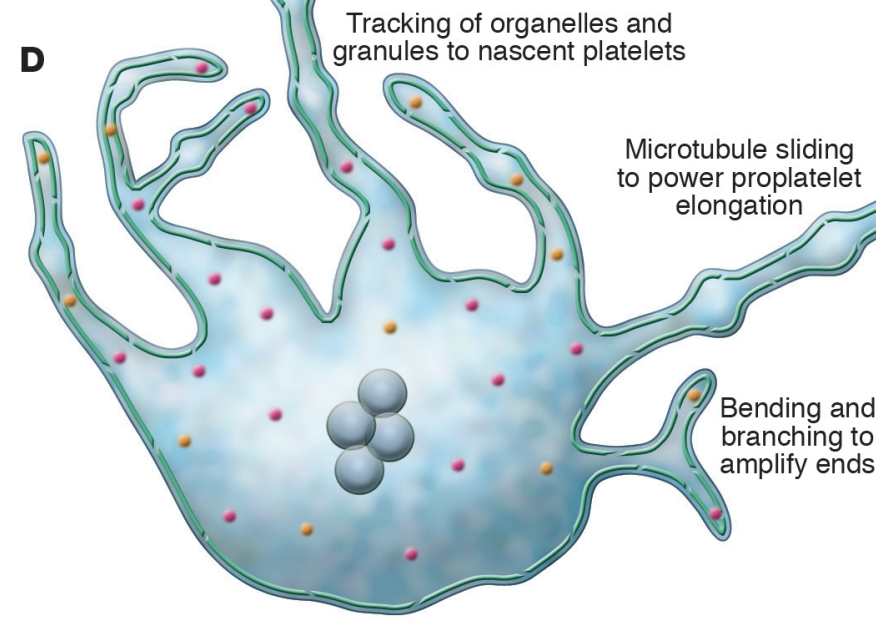

Tracking of organelles and
granules to nascent platelets

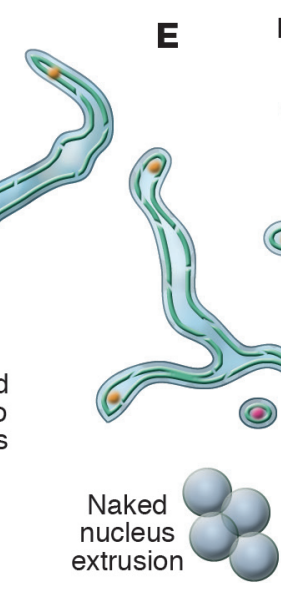

\section{Figure 4}

Overview of megakaryocyte production of platelets. As megakaryocytes transition from immature cells (A) to released platelets (E), a systematic series of events occurs. (B) The cells first undergo nuclear endomitosis, organelle synthesis, and dramatic cytoplasmic maturation and expansion, while a microtubule array, emanating from centrosomes, is established. (C) Prior to the onset of proplatelet formation, centrosomes disassemble and microtubules translocate to the cell cortex. Proplatelet formation commences with the development of thick pseudopods. (D) Sliding of overlapping microtubules drives proplatelet elongation as organelles are tracked into proplatelet ends, where nascent platelets assemble. Proplatelet formation continues to expand throughout the cell while bending and branching amplify existing proplatelet ends. (E) The entire megakaryocyte cytoplasm is converted into a mass of proplatelets, which are released from the cell. The nucleus is eventually extruded from the mass of proplatelets, and individual platelets are released from proplatelet ends.

Results from studies on GPIb $\alpha$ knockout mice, a model of Bernard-Soulier syndrome, indicate that the megakaryocyte DMS is disordered (70). These megakaryocytes went on to produce aberrantly large megakaryocyte fragments and proplatelets with reduced internal membrane content (71). Since the GPIb/IX/V complex is linked to the membrane skeleton, it is thought that the genetic defects of Bernard-Soulier syndrome may alter normal cytoskeletal dynamics during platelet formation (69).

MYH9-related disorders. MYH9-related disorders are characterized by macrothrombocytopenia. Patients with these disorders, including May-Hegglin anomaly, Sebastian syndrome, Fechtner syndrome, Alport syndrome, and Epstein syndrome, may also experience hearing loss, cataract, nephritis, and/or granulocyte inclusion body formation (69). The genetic defects in each of these illnesses occur within the MYH9 gene, which encodes nonmuscle myosin heavy chain IIA, the sole myosin isoform expressed in platelets and neutrophils. Myosin molecules are hexameric protein complexes composed of 2 heavy chains that dimerize and 2 pairs of myosin light chains. Each heavy chain consists of a globular head containing ATPase activity and actin-binding sites, and an $\alpha$-helical tail or rod. Although numerous mutations have been identified throughout the MYH9 gene, mutations are most commonly seen to occur in exons encoding the $\alpha$-helical tail, a region that promotes myosin dimerization and filament formation. Studies examining the 4 most common myosin rod mutations identified in MYH9-related disorders indicate that the mutations result in diminished myosin dimer and filament formation in vitro (72). Moreover, studies completed on 2 MYH9 mutations that reside within the myosin head domain reveal that the defects dramatically decrease both the MgATPase activity and actin filament translocation of myosin in vitro (73).

The thrombocytopenia that occurs in MYH9-related disorders is thought to be a result of defective platelet production, since 
both megakaryocyte numbers and platelet clearance are normal (74). Myosin has been theorized to function in platelet formation, specifically during the bending and branching process, where an actomyosin interaction could possibly provide the forces required to contort the proplatelet shaft (27). Also, recent studies on platelets carrying MYH9 defects show that a greater amount of myo$\sin$ is associated with the actin cytoskeleton in resting platelets and that, upon activation, MYH9-mutated platelets have altered cytoskeletal dynamics (75). Defects in surface expression of the GPIb/IX/V complex in platelets carrying MYH9 mutations have also been reported (76).

Gray platelet syndrome. Gray platelet syndrome is another autosomal dominant disease that presents with macrothrombocytopenia. These large platelets appear gray due to a reduction in $\alpha$-granule content (77). $\alpha$-granules normally contain a number of proteins including von Willebrand factor, and fibrinogen. In Gray platelet syndrome, platelets inadequately package these proteins within $\alpha$-granules (78). As a consequence, a number of clot promoting factors fail to be released upon platelet activation, which increases the risk of bleeding. Although the precise genetic defects responsible for gray platelet syndrome are unknown, evidence indicates that cytoskeletal defects can result in poor $\alpha$-granule packaging (79).

\section{Conclusions}

The transition from megakaryocyte to platelets is a complex process. Although the basic mechanisms of platelet production have been investigated, elucidating the specific molecular controls and cellular events involved in platelet formation and release is an unfinished task. Major issues still to be addressed include (a) determination of which factors induce proplatelet formation in mature megakaryocytes, (b) identification of the mechanism of platelet release from proplatelets, and (c) understanding of how the cytoskeleton drives the events that result in proplatelet production. Further examination of genetic defects that result in platelet disorders, in addition to continued molecular, cellular, and biochemical studies of megakaryocytes as they transition into platelets, will provide a clearer understanding of these processes. This knowledge may aid in the future ex vivo expansion of platelets or in vivo therapies aimed at enhancing platelet production in patients with thrombocytopenia.

\section{Acknowledgments}

This work was supported by NIH grant HL68130 (to J.E. Italiano Jr.). J.E. Italiano Jr. is an American Society of Hematology Scholar. S.R. Patel was supported by NIH training grant HL066978-04 and an NIH/National Heart, Lung, and Blood Institute postdoctoral fellowship award (HL082133).

Address correspondence to: Joseph E. Italiano Jr., Brigham and Women's Hospital, Division of Hematology, 1 Blackfan Circle, 6th Floor, Boston, Massachusetts 02115, USA. Phone: (617) 355-9007; Fax: (617) 355-9016; E-mail: jitaliano@rics.bwh.harvard.edu.
1. Ogawa, D. 1993. Differentiation and proliferation of hematopoietic stem cells. Blood. 81:2844-2853.

2. Briddell, R., Brandt, J., Stravena, J., Srour, E., and Hoffman, R. 1989. Characterization of the human burst-forming unit-megakaryocyte. Blood. 59:145-151.

3. Kaushansky, K. 2005. The molecular mechanisms that control thrombopoiesis. J. Clin. Invest. 115:3339-3347. doi:10.1172/JCI26674.

4. Kaushansky, K., and Drachman, J.G. 2002. The molecular and cellular biology of thrombopoietin: the primary regulator of platelet production. Oncogene. 21:3359-3367.

5. Long, M., Williams, N., and Ebbe, S. 1982. Immature megakaryocytes in the mouse: physical characteristics, cell cycle status, and in vitro responsiveness to thrombopoietic stimulatory factor. Blood. 59:569-575.

6. Odell, T.T., Jr., Jackson, C.W., and Reiter, R.S. 1968. Generation cycle of rat megakaryocytes. Exp. Cell Res. 53:321-328.

7. Ebbe, S. 1976. Biology of megakaryocytes. Prog. Hemost. Thromb. 3:211-229.

8. Ebbe, S., and Stohlman, F. 1965. Megakaryocytopoiesis in the rat. Blood. 26:20-34.

9. Ravid, K., Zimmet, J.M., and Jones, M.R. 2002. Roads to polyploidy: the megakaryocyte example. J. Cell. Physiol. 190:7-20.

10. Nagata, N., Muro, Y., and Todokoro, K. 1997. Thrombopoietin-induced polyploidization of bone marrow megakaryocytes is due to a unique regulatory mechanism in late mitosis. J. Cell Biol. 139:449-457.

11. Vitrat, N., et al. 1998. Endomitosis of human megakaryocytes is due to abortive mitosis. Blood. 91:3711-3723.

12. Odell, T., Jackson, C., and Friday, T. 1970. Megakaryocytopoiesis in rats with special reference to polyploidy. Blood. 35:775-782.

13. Yamada, F. 1957. The fine structure of the megakaryocyte in the mouse spleen. Acta Anat. (Basel). 29:267-290.

14. Behnke, O. 1968. An electron microscope study of megakaryocytes of rat bone marrow. I. The development of the demarcation membrane system and the platelet surface coat. J. Ultrastruct. Res. 24:412-433.

15. Nakao, K., and Angrist, A. 1968. Membrane surface specialization of blood platelet and megakaryocyte. Nature. 217:960-961.

16. Gerrard, J., White, J., Rao, G., and Townsend, D. 1976. Localization of platelet prostaglandin production in the platelet dense tubular system. Am.J. Pathol. 101:353-364.

17. Henijnen, H.F., et al. 1998. Multivesicular bodies are an intermediate stage in the formation of platelet alpha granules. Blood. 91:2313-2325.

18. Handagama, P.J., George, M., Shuman, R., McEver, R., and Bainton, D.F. 1987. Incorporation of circulating protein into megakaryocyte and platelet granules. Proc. Natl. Acad. Sci. U. S. A. 84:861-865.

19. Radley, J.M., and Haller, J.C. 1982. The demarcation membrane system of the megakaryocyte: a misnomer? Blood. 60:213-219.

20. Wright, J. 1906. The origin and nature of blood platelets. Boston Med. Surg. J. 154:643-645.

21. Becker, R.P., and DeBruyn, P.P. 1976. The transmural passage of blood cells into myeloid sinusoids and the entry of platelets into the sinusoidal circulation: a scanning electron microscopic investigation. Am. J. Anat. 145:183-205.

22. Thiery, J., and Bessis, M. 1956. Genesis of blood platelets from the megakaryocytes in living cells [In French]. C. R. Hebd. Seances Acad. Sci. 242:290-292.

23. Behnke, O. 1969. An electron microscope study of the rate megakaryocyte. II. Some aspects of platelet release and microtubules. J. Ultrastruct. Res. 26:111-129.

24. Kaushansky, K. 2003. Thrombopoietin: a tool for understanding thrombopoiesis. J. Thromb. Haemost. 1:1578-1592.

25. Leven, R.M., and Nachmias, V.T. 1982. Cultured megakaryocytes: changes in the cytoskeleton after ADP-induced spreading. J. Cell Biol. 92:313-323.

26. Tablin, F., Castro, M., and Leven, R.M. 1990. Blood platelet formation in vitro. The role of the cytoskeleton in megakaryocyte fragmentation. J. Cell
Sci. 97:59-70

27. Italiano, J.E., Lecine, P., Shivdasani, R., and Hartwig, J.H. 1999. Blood platelets are assembled principally at the ends of proplatelet processes produced by differentiated megakaryocytes. J. Cell Biol. 147:1299-1312.

28. Choi, E., Nichol, J.L., Hokom, M.M., Hornkohl, A.C., and Hunt, P. 1995. Platelets generated in vitro from proplatelet-displaying human megakaryocytes are functional. Blood. 85:402-413.

29. Cramer, E., et al. 1997. Ultrastructure of platelet formation by human megakaryocytes cultured with Mpl ligand. Blood. 89:2336-2346.

30. Tavassoli, M., and Aoki, M. 1989. Localization of megakaryocytes in the bone marrow. Blood Cells. 15:3-14.

31. Scurfield, G., and Radley, J.M. 1981. Aspects of platelet formation and release. Am. J. Hematol. 10:285-296.

32. Lichtman, M., Chamberlain, J., Simon, W., and Santillo, P. 1978. Parasinusoidal location of megakaryocytes in marrow: a determinant of platelet release. Am. J. Hematol. 4:303-312.

33. Crispino, J.D. 2005. GATA-1 in normal and malignant hematopoiesis. Semin. Cell Dev. Biol. 16:137-147.

34. Shivdasani, R.A., Fujiwara, Y., McDevitt, M.A., and Orkin, S.H. 1997. A lineage-selective knockout establishes the critical role of transcription factor GATA-1 in megakaryocyte growth and platelet development. EMBOJ. 16:3965-3973.

35. Mehaffey, M.G., Newton, A.L., Gandhi, M.J., Crossley, M., and Drachman, J.G. 2001. X-linked thrombocytopenia caused by a novel mutation of GATA-1. Blood. 98:2681-2688.

36. Andrews, N.H., Erdjument-Bromage, H., Davidson, M., Tempest, P., and Orkin, S. 1993. Erythroid transcription factor NF-E2 is a hematopoietic-specific basic-leucine zipper protein. Nature. 362:722-728.

37. Shivdasani, R.A., et al. 1995. Transcription factor NF-E2 is required for platelet formation independent of the actions of thrombopoietin/MGDF in megakaryocytes. Cell. 81:695-704. 
38. Lecine, P., et al. 1998. Mice lacking transcription factor NF-E2 provide in vivo validation of the proplatelet model of thrombopoiesis and show a platelet production defect that is intrinsic to megakaryocytes. Blood. 92:1608-1616.

39. Lecine, P., Italiano, J.E., Kim, S., Villeval, J., and Shivdasani, R. 2000. Hematopoietic-specific beta 1 tubulin participates in a pathway of platelet biogenesis dependent on the transcription factor NF-E2. Blood. 96:1366-1373.

40. Tiwari, S., et al. 2003. A role for Rab27b in NF-E2dependent pathways of platelet formation. Blood. 102:3970-3979.

41. Deveaux, S., et al. 1997. p45 NF-E2 regulates expression of thromboxane synthase in megakaryocytes. ЕMBO J. 16:5654-5661.

42. Kerrigan, S.W., Gaur, M., Murphy, R.P., Shattil, S.J., and Leavitt, A.D. 2004. Caspase 12: a developmental link between G-protein-coupled receptors and integrin alphaIIbeta3 activation. Blood. 104:1327-1334.

43. Topp, K.S., Tablin, F., and Levin, J. 1990. Culture of isolated bovine megakaryocytes on reconstituted basement membrane matrix leads to proplatelet process formation. Blood. 76:912-924.

44. Schwer, H.P., Lecine, P., Tiwari, S., Italiano, J.E., and Hartwig, J. 2001. A lineage-restricted and divergent beta-tubulin isoform is essential for the biogenesis, structure and function of blood platelets. Curr. Biol. 11:579-589.

45. Schulze, H., et al. 2004. Interactions between the megakaryocyte/platelet-specific beta 1 tubulin and the secretory leukocyte protease inhibitor SLPI suggest a role for regulated proteolysis in platelet functions. Blood. 104:3949-3957.

46. Handagama, P.J., Feldman, B.F., Jain, N.C., Farver, T.B., and Kono, C.S. 1987. In vitro platelet release by rat megakaryocytes: effect of metabolic inhibitors and cytoskeletal disrupting agents. Am. J. Vet. Res. 48:1142-1146.

47. Freson, K., et al. 2005. The TUBB1 Q43P functional polymorphism reduces the risk of cardiovascular disease in men by modulating platelet function and structure. Blood. 106:2356-2362.

48. Italiano, J.E., Shivdasani, R.A., and Hartwig, J.H. 2002. Cytoskeletal mechanics of platelet formation [abstract]. Blood. 100:132a.

49. Patel, S.R., et al. 2005. Differential roles of microtubule assembly and sliding in proplatelet formation by megakaryocytes. Blood. doi:10.1182/blood2005-06-2204.

50. Trowbridge, E., et al. 1984. The origin of platelet count and volume. Clin. Phys. Physiol. Meas. 5:145-156.
51. Harker, L., and Finch, C. 1969. Thrombokinetics in man. J. Clin. Invest. 48:963-974.

52. Kaufman, R., Airo, R., Pollack, S., and Crosby, W. 1965. Circulating megakaryocytes and platelet release in the lung. Blood. 26:720-731.

53. Rojnuckarin, P., and Kaushansky, K. 2001. Actin reorganization and proplatelet formation in murine megakaryocytes: the role of protein kinase C alpha. Blood. 97:154-161.

54. Richardson, J., Shivdasani, R., Boers, C., Hartwig, J., and Italiano, J.E. 2005. Mechanisms of organelle transport and capture along proplatelets during platelet production. Blood. doi:10.1182/blood2005-06-2206.

55. Gordge, M.P. 2005. Megakaryocyte apoptosis: sorting out the signals. Br. J. Pharmacol. 145:271-273.

56. Radley, J.M., and Haller, J.C. 1983. Fate of senescent megakaryocytes in the bone marrow. Br. J. Haematol. 53:277-287.

57. Falcieri, E., et al. 2000. Ultrastructural characterization of maturation, platelet release and senescence of human cultured megakaryocytes. Anat. Rec. 258:90-99.

58. Zauli, G., et al. 1997. In vitro senescence and apoptotic cell death of human megakaryocytes. Blood. 90:2234-2243.

59. Kaluzhny, Y., and Ravid, K. 2004. Role of apoptotic processes in platelet biogenesis. Acta Haematol. 111:67-77.

60. De Botton, S., et al. 2002. Platelet formation is the consequence of caspase activation within megakaryocytes. Blood. 100:1310-1317.

61. Kaluzhny, Y., et al. 2002. BclxL overexpression in megakaryocytes leads to impaired platelet fragmentation. Blood. 100:1670-1678.

62. Sanz, C., et al. 2001. Antiapoptotic protein Bcl-xL is up-regulated during megakaryocytic differentiation of CD34(+) progenitors but is absent from senescent megakaryocytes. Exp. Hematol. 29:728-735.

63. Battinelli, E., and Loscalzo, J. 2000. Nitric oxide induces apoptosis in megakaryocytic cell lines. Blood. 95:3451-3459.

64. Battinelli, E., Willoughby, S.R., Foxall, T., Valeri, C.R., and Loscalzo, J. 2001. Induction of platelet formation from megakaryocytoid cells by nitric oxide. Proc. Natl. Acad. Sci. U. S. A. 98:14458-14463.

65. Kim, J.A., et al. 2002. Gene expression profile of megakaryocytes from human cord blood CD34+ cells ex vivo expanded by thrombopoietin. Stem Cells. 20:402-416.

66. Clarke, M.C., Savill, J., Jones, D.B., Nobel, B.S., and Brown, S.B. 2003. Compartmentalized megakaryocyte death generates function platelets com- mitted to caspase-independent death. J. Cell Biol. 160:577-587.

67. Brown, S.B., Clarke, M.C., Magowan, L., Sanderson, H., and Savill, J. 2000. Constitutive death of platelets leading to scavenger receptor-mediated phagocytosis. A caspase-independent cell clearance program. J. Biol. Chem. 275:5987-5996.

68. Bernard, J., and Soulier, J.P. 1948. Sur une nouvelle variet e de dystrophie thrombocytarie-hemorragipare congenitale. Sem. Hop. Paris. 24:3217-3223.

69. Balduini, C.L., and Savoia, A. 2002. Inherited thrombocytopenias: from genes to therapy. Haematologica. 87:860-880.

70. Ware, J., Russel, S., and Ruggeri, Z.M. 2000. Generation and rescue of a murine model of platelet dysfunction: the Bernard-Soulier syndrome. Proc. Natl. Acad. Sci. U. S. A. 97:2803-2808.

71. Poujol, C., Ware, J., Nieswandt, B., Nurden, A.T., and Nurden, P. 2002. Absence of GPIbalpha is responsible for aberrant membrane development during megakaryocyte maturation: ultrastructural study using a transgenic model. Exp. Hematol. 30:352-360.

72. Franke, J.D., Dong, F., Rickoll, W.L., Kelley, M.J., and Kiehart, D.P. 2004. Rod mutations associated with MYH9-related disorders disrupt non-muscle myosin-IIA assembly. Blood. 105:161-169.

73. Hu, A., Wang, F., and Sellers, J.R. 2002. Mutations in human nonmuscle myosin IIA found in patients with May-Hegglin anomaly and Fechtner syndrome result in impaired enzymatic function. J. Biol. Chem. 277:46512-46517.

74. Hamilton, R.W., et al. 1980. Platelet function, ultrastructure, and survival in the May-Hegglin anomaly. Am. J. Clin. Pathol. 74:663-668.

75. Canobbio, I., Noris, P., Pecci, A., Balduini, C.L., and Torti, M. 2005. Altered cytoskeleton organization in platelets from patients with MYH9-related disease. J. Thromb. Haemost. 3:1026-1035.

76. DiPumpo, M., et al. 2002. Defective expression of GPIb/IX/V complex in platelets from patients with May-Hegglin anomaly and Sebastian syndrome. Haematologica. 87:943-947.

77. Raccuglia, G. 1971. Gray platelet syndrome. Am. J. Med. 51:818-828.

78. Oramer, E.M., Vainchenker, W., Vinci, G., Guichard, J., and Breton-Gorius, J. 1985. Gray platelet syndrome: immunoelectron microscopic localisation of fibrinogen and von Willebrand factor in platelets and megakaryocytes. Blood. 66:1309-1316.

79. Stenberg, P.E., et al. 1998. Prolonged bleeding time with defective platelet filopodia formation in the Wistar Furth rat. Blood. 91:1599-1608. 